\title{
USING TEXTURE AND SHAPE FEATURES TO RETRIEVE SETS OF SIMILAR MEDICAL IMAGES
}

\author{
JiAnN-Der LeE, Li-Peng Lou
}

Department of Electrical Engineering

Chang Gung University, Tao-Yuan, Taiwan

\begin{abstract}
In this paper, a novel scheme has been proposed for image retrieval task using the feature extracted directly from a compressed or uncompressed image. The texture information is first extracted by exploiting the multiresolution nature of wavelet decomposition, which represent the horizontal, vertical and diagonal frequency distribution of an image. We then calculate the mean and standard deviation of wavelet coefficients of each sub-band as texture features. In additions, we also extract shape feature by using the fixed-resolution block representation, which divides the image into isometric blocks and calculate the overlapped degree of each block with binary codes. The experimental results show that the retrieval efficiency is considerably improved by the proposed approach.
\end{abstract}

Biomed Eng Appl Basis Comm, 2003 (October); 15: 193-199.

Keywords: Image retrieval, shape feature, texture feature, similar medical image

\section{INTRODUCTION}

The rapid development of computer network has produced many convenient applications. For example, in modern hospital, the patient record is made electronically and applied with various recourses in teleconsultation. Many medical images, i.e. ultrasonography, CT (computerized tomography) and $\mathrm{MRl}$ (magnetic resonance image), are used to diagnose the patient's status in the hospitals. Thus there are large numbers of films for storing medical image in the hospitals. Now by PACS (Picture Archiving and Communication System), the whole image diagnosis system in the hospital can be integrated, the medical images can be digitized, and the medical image database can be built, so as to make it convenient for

Received: Sept 17, 2003; Accepted: Oct 1, 2003 Correspondence: Jiann-Der Lee, Ph. D., Professor Department of Electrical Engineering, Chang Gung University, Tao-Yuan, Taiwan

E-mail: jdlee@mail.cgu.edu.tw diagnosing the patients and inquiring about the relative illness. An image needs many memory bits for store, for example, a digital image with $256 \times 256$ pixels and 256 gray levels needs the storage of 65536 bytes. Confronted with the increasingty numerous and various medical images, the users want to find simply and rapidly the similar medical image of a certain interested illness for diagnosis and analysis; it is a big problem at present.

In general, there are two kinds of features common used to represent the image contents. One is obtained in the spatial domain, the objective characteristics of image pixel distribution, i.e. object shape [1], texture characteristic [2], color distribution [3-4], and other image contents, are used to represent the characteristic of image. The other is obtained in the frequency domain, because some images have more distinct features in frequency domain than that in spatial domain; therefore, the results of some transforms, i.e. Fast Fourier Transform [5], Discrete Cosine Transform [6] or Discrete Wavelet Transform [7-8], can be recorded as the features of image. Most relative researches combine some different image features, i.e. relation of color and texture [9], color and 
shape [10] or color and space [11], etc, by some comparison methods to abstract the features of various images as the basis for image retrieval, so that the image of the maximum similarity can be found out.

Besides, whether the size of image data will cause network jam during data transfer and the space required for saving the image data are also problems; therefore, the image data should be compressed. The so-called image compression is reducing the redundancy of image data, including psychological, inter-image pixel and code redundancy, and leaving over the data that we are interested in. JPEG is the image compression standard that are adopted most widcly at present, and the newly constituted JPEG2000 will be the standard format for image compression in the coming $21^{\text {st }}$ century. Recently, Cheng [12] proposed a retrieval method aiming at the uncompressed and compressed JPEG or the Wavelet Transformed image. Extension of the previous researches, in this paper, we not only proposed a novel image retrieval scheme suitable for various image format including BMP, JPEG and JPEG2000, but also made retricval aiming at the whole similar compressed medical image set to find out the features of image that can represent thc whole image set; hercin, the users only have to find out which set that the image belongs to before he compare which image it is. The objective that we hoped to achieve is the image retrieval system that can reduce the quantity of image data and computation, and enhance the correctness and effectiveness of image access and retrieval.

As shown in Fig. 1, this paper presents a contentbased image retrieval strategy, in expectation of finding out the features of shape and texture from all the compressed data; therefore, a composite image retrieval scheme is built as the model for retrieval of the similar compressed medical image set, which extracts the features image from the medical images of different format in the whole set to represent the original image and then finds out the features of shape and texture from the feature image for comparison. From the experimental results, it is proved that the proposed method can effectively complete the task of image retrieval.

The remainder of this paper is organized as below. Section 2 illustrates the proposed image retrieval strategy, which describes how to classify the images into the corresponding sets according to their correlation coefficients. The method to extract the texture and shape feature of a feature image is also included in this section. The experimental results are shown in Section 3. Finally, the conclusion is given in Section 4.

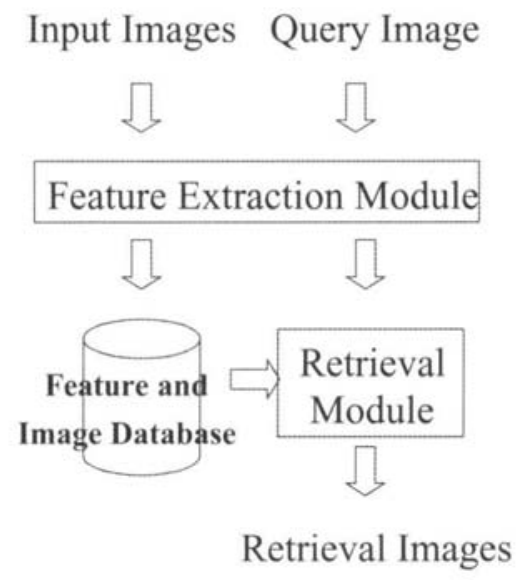

Fig. 1 The flowchart of the proposed scheme for image retrieval.

\section{IMAGE RETRIEVAL STRATEGY}

In this paper, the major retrieval data are the similar medical image sets; most of these images are the simple combination of object and background. Therefore, on consideration of the above objectives and the practical application, we designed an image retrieval strategy, which made retricval of the medical image separately aiming at the whole set of different formats including uncompressed and compressed JPEG or JPEG 2000 using texture and shape features extracted from medical images. The flowchart of the retrieval strategy is shown in Fig.1.

\subsection{Classification Method of Similar Images}

Taking the medical image as an example, when taking CT and MRI scanning, it usually takes tens of pieces once. If the inter-distance between the adjacent images is too large, the difference between them will be too large and they cannot be considered as similar images; therefore, we defined a simple criterion to classify the images. That is, based on the statistic relation of images, we can calculate their correlation coefficient and assign each image to its corresponding category.

Supposing there are two pieces of image data, which are $A-\left(a_{1}, a_{2}, \ldots a_{1}\right)$ and $B-\left(b_{1}, b_{2}, \ldots b_{4}\right)$ separately, therefore the linear correlation coefficient of the two images is written as Eq.(1).

$$
r_{A, B}=\frac{\sum_{i=1}^{S}\left(a_{i}-a_{m}\right)\left(b_{i}-b_{m}\right)}{\sqrt{\sum_{i=1}^{S}\left(a_{i}-a_{m}\right)^{2}} \sqrt{\sum_{i=1}^{S}\left(b_{i}-b_{m}\right)^{2}}}
$$


Herein, $a_{m}$ and $b_{m}$ represent the average value of $A$ and $B$ data sets, respectively; $S$ is the total pixel number of an image. In order to make it convenient for observation and avoid producing negatives, we replaced $\gamma_{A . B}$ with $\gamma_{A, B}^{2}$. Therefore, if the two images are very similar, $r_{\text {A.B }}^{2}$ will tend towards 1 ; on the contrary, if the two images have no relativity, $r^{2}{ }_{A, B}$ nearly equals to 0 .

Supposing there is a set of successive medical images $I_{1}, I_{2}, I_{3} \ldots I_{N}$, the correlation coefficients between $I_{1}$ and $I_{2}, I_{1}$ and $I_{3} \ldots I_{1}$ and $I_{j+1}$ are calculated; if the correlation cocfficient is smaller than a certain threshold, we will classify the images from $I_{1}$ to $I_{\text {, }}$ as the same category. Then the correlation coefficients between $I_{j+1}$ and $I_{j+2}, I_{j-1}$ and $I_{j, 3} \ldots I_{j, 1}$ and $I_{k}$ are calculated; if the correlation coefficient between $I_{j+1}$ and $I_{k+1}$ is smaller than a certain threshold, the images from $\mathrm{I}_{\mathrm{j}-1}$ to $I_{k}$ are classified as the same category; accordingly, the images can be classified correctly.

\subsection{Representing the Whole Image Set with Centroid Image}

The so-called Centroid Method [13] extracts the pixels in the same position of each image of an image set and sorts them in order; thus the centroid image of the similar image set can be found out. The Centroid Method but not Average method is adopted because it can avoid the effect caused by huge variation of the gray level among the images in an image set; In other words, it can remove the extreme gray value due to the noise. Fig. 2 shows the flowchart of Centroid Method.

\subsection{Representing Images of Different Formats with Feature Image}

Because the computation of feature information for the whole image set is too huge, in order to reduce the computation of these image features, we attempt to replace the whole set of images by a feature image without affecting the retrieval accuracy. At the same time the feature image can be sorted out from images of different formats, including compressed and uncompressed images, then the image features of the feature image is recorded as the retrieval index. Various image formats used in this approach are described as below:

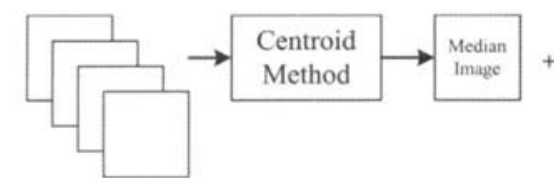

Set of similiar Images

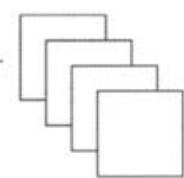

difference Images
Fig. 2 The flowchart of Centroid Method.

\subsubsection{Original Image (BMP)}

The image with BMP format is first divided into blocks of $8 \times 8$ pixels, and the average value of the blocks is calculated as a pixel of the feature image. That is, we reduce a $512 * 512$ to a $64 * 64$ feature image. The detailed formula is expressed as Eq. (2).

$$
F I_{m, n}=\frac{1}{64} \sum_{i=0}^{7} \sum_{j=0}^{7} O I_{8 m+i, 8 n+j}
$$

where $F I_{m, n}$ represents the pixel of coordinate $(m, n)$ in the feature image, OI represents the original image.

\subsubsection{Compressed Image (JPEG)}

The image with JPEG format is also divided into blocks in advance and Discrete Cosine Transform (DCT) are performed to these blocks. The DC coefficient and $\mathrm{AC}$ coefficient of these blocks after DCT are then coded separately; therefore, DC coefficient can be adopted as the representative image during image retrieval. In order to make the feature image found out from JPEG similar with that found out from original BMP image, we multiply the DC coefficient of number $(m, n)$ block of the image by $(1 / 8)$ plus 128 , i.e., $\left[(1 / 8) \cdot F I_{m . n}(0,0)+128\right], 128$ is added back because it is subtracted from the image in JPEG compression as displacement. In short, the JPEG format image is divided into $8 * 8$ blocks and the calculation step is summarized as below.

Supposing $F_{m, n}(x, y)$ is the value of number $(m, n)$ block of the image after Fast Fourier Transform, therefore DC coefficient is $F_{m . n}(0,0)$, among which $\mathrm{N}=8$.

$$
\begin{aligned}
F_{m, i}(0,0) & =\frac{1}{\sqrt{8}} \cdot \frac{1}{\sqrt{8}} \sum_{i=0}^{N-1} \sum_{j=0}^{k-1} O L_{8 m+, 8 n,} \cdot \cos \left(\frac{(2 x+1) \cdot 0 \cdot \pi)}{2 N}\right) \cos \left(\frac{(2 y+1) \cdot 0 \cdot \pi}{2 N}\right) \\
& =\frac{1}{8} \sum_{i=0}^{N} \sum_{j=0}^{N} O I_{8 m+1,8 n+j} \\
& =8 \cdot \frac{1}{64} \sum_{i=0}^{7} \sum_{j=0}^{7} O I_{8 m+i, 8 n+j} \\
& =8 \cdot \mathrm{FI}_{\mathrm{m}, \mathrm{n}}
\end{aligned}
$$

\subsubsection{Compressed Image (JPEG2000)}

In order to achicve the goal that the feature image obtained from images with various formats should be as same as possible, the derivation of the feature image 
from a compressed image (JPEG2000) is described herer. It is known that the message will be multiplied by 2 when it is made number i step two-dimensional Wavelet Transform. Therefore, in order to find out the representative image from JPEG2000 similar with that from the original image, we divide $2^{1}$ from $L_{L}$ of number $(m, n)$ block plus displacement (DC), so that we can get the approximate average value of the image using Eq. (4).

$$
F I_{m, n}=\frac{L L_{i}}{2^{i}}+D C
$$

\subsection{Extracting Image Features from the Feature Image}

In order to find out the feature that is sufficient to represent the whole set of image, first we analyze the content characteristic of image and extract the characteristics of its shape and texture, etc, and then combine these two features for later retrieval task.

\subsubsection{Texture-based Feature Extraction}

The image is calculated by three-level Discrete Wavelet Transform and then sub-images are obtained. Nine sets of Wavelet coefficients, including $\mathrm{LH}_{1}, \mathrm{HL}$, $\mathrm{HH}_{1}, \mathrm{LH}_{2}, \mathrm{HL}_{2}, \mathrm{HH}_{2}, \mathrm{LH}_{3}, \mathrm{HL}_{3}, \mathrm{HH}_{3}$, are taken out. Their mean and standard deviation [12] are calculated as image features, as Eq. (5) and (6).

$$
\text { Mean } M_{\imath}=\frac{1}{W \cdot H} \sum_{x=0}^{W-1} \sum_{y=0}^{H-1}\left(S_{t}(x, y)\right)
$$

where $\mathrm{Si}$ represents the i step wavelet coefficient, $\mathrm{i}=1,2,3$.

Standard deviation

$$
\sigma_{i}=\sqrt{\iint\left(\left|S_{i}(x, y)\right|-M_{i}\right)^{2} d x d y}
$$

The feature vector is then obtained from the mean and standard deviation as follows

$$
f=\left[M_{0}, \sigma_{0}, M_{1}, \sigma_{1}, M_{2}, \sigma_{2}, \ldots . ., M_{10}, \sigma_{10}\right]
$$

After the feature vector of image is obtained, the distance between image $I_{1}$ and $I_{z}$ is calculated.

$$
\operatorname{dist}\left(I_{1}, I_{2}\right)=\sum_{k=0}^{1}\left|f_{k}^{\prime_{1}}-f_{k}^{\prime=}\right|
$$

$f_{z}^{I_{1}}$ and $f_{k}^{I_{2}}$ represent the $\mathrm{k}^{\text {dh }}$ features of image $\mathrm{I}_{1}$ and

\subsubsection{Shape-based Feature Extraction}

Here, we uses Fixed-Resolution format for shape representation and derive the required shape features. The details of the extraction step are described as below.

Step 1: Divide the image into isometric blocks that contain $\mathrm{N} \times \mathrm{N}$ pixels.

Step 2: Judge whether there are over $p \%$ (p is between 1 100) pixels greater than a certain critical value in each block; if it is true, the index of this block will be set to 1 , if it is not, it will be set to 0 .

Step 3: Judge whether the shapes of the two objects are similar; comparing the block index produced in step 2 , if they are different, then add 1 to the result; the smaller result represents that the shapes arc more similar. In short, the decision rule is shown as below:

$$
\begin{aligned}
& \text { 1. if } O I_{m, n}(i, j)>\text { threshold, } \quad F_{m, n}(i, j)=1 \\
& \text { else } F_{m, n}(i, j)=0 \\
& \text { 2. if } \sum_{i=1}^{n} \sum_{j=1}^{n} F_{m, n}(i, j)>N^{2} \cdot P \% \text {, } \\
& \operatorname{Index}(m, n)=1 \quad \text { else } \quad \operatorname{Index}(m, n)=0 \\
& \text { 3. } \quad \text { dist }=\sum_{m=1}^{N} \sum_{n-1}^{*}|\operatorname{Index}(m, n)-q \operatorname{Index}(m, n)|
\end{aligned}
$$

For example: An image with $256 \times 256$ pixels, if $\mathrm{N}$ equals to 32 , the image is divided into $8 \times 8$ blocks, each block contains $32 \times 32$ pixels; if $\mathrm{N}$ equals to 16 , the image is divided into $16 \times 16$ blocks, each block contains $16 \times 16$ pixels. Therefore, if there are more blocks, in spite of more data quantity, more specific object shape can be recorded and the resolution is higher. Fig. 3 is an example to illustrate the concept of shape representation using Fixed-Resolution format.

\section{EXPERIMENTAL RESULTS AND PERFORMANCE EVALUATION}

In the experiment, a medical image database, which consists of MRI images and CT images of human body partially obtained from Chang Gung Memorial Hospital, are employed. Six examples of the test images are shown in Fig. 4. More specifically, MRI images include the six principal parts of human body; each part has several similar image sets, totaled to 580 pieces; and the resolution of each image is 256 


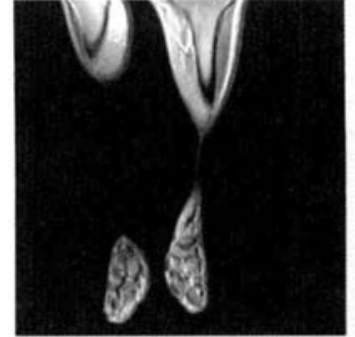

(a)

(b)

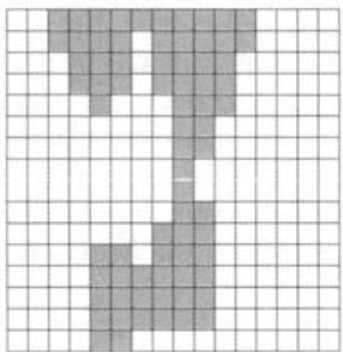

Fig. 3 An example to illustrate the concept of shape representation using Fixed-Resolution format. (a) a source image, (b) the shape representation of (a) with the block size $32 \times 32$ pixels, (c) the shape representation of (a) with the block size $16 \times 16$ pixels.

x 256 pixels with 8 bits/pixels. We classify the whole set of images into 145 sets, each has 4 slices, by using their correlation coefficients. The element of this MRI image database is illustrated as follow:

1. Head, 64 slices, totaled to 16 sets.

2. Shoulder, 116 slices, totaled to 29 sets.

3. Trunk, 128 slices, totaled to32sets.

4. Thigh, 108 slices, totaled to 27 sets.

5. Legs, 68 slices, totaled to 17 sets.

6. Feet, 96 slices, totaled to 24 sets.

The other source of the used image database is CT images. Each of CT images has $256 \times 256$ pixels with $8 \mathrm{bits} /$ pixels, including a set of head images with 49 slices and a set of chest images with 15 slices.

To evaluate the performance of the proposed scheme for image retrieval, we use the common used factor, i.e. Precision and Recall, to represent the retrieval accuracy.

Supposing the retrieval image is one of the images stored in the database, we define the accuracy to be $100 \%$ if the first candidate image provided by the system is just the desired image; otherwise, the accuracy is $0 \%$. Consequently, we want to know the percentage of times that the images are correctly

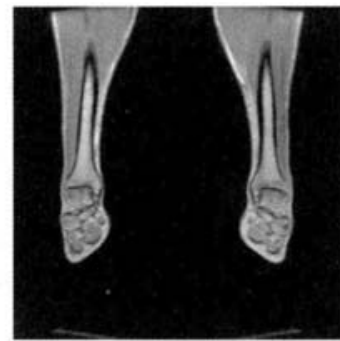

(a)

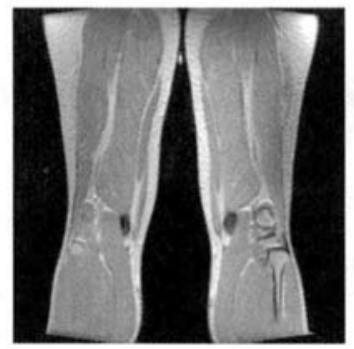

(c)

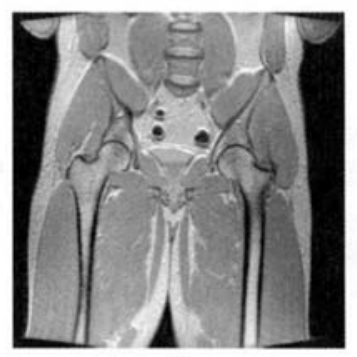

(e)

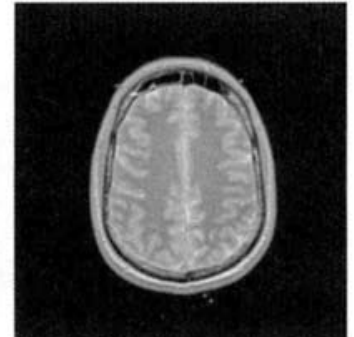

(b)

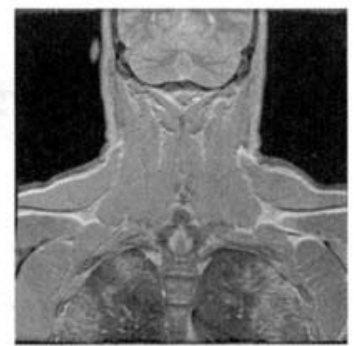

(d)

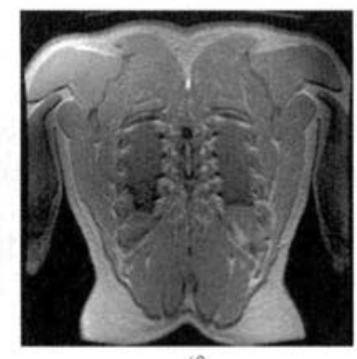

Fig. 4 Six examples of test images used in the experiment.

retrieved in the total retrieval times. Based on this concept, the factor Precision is defined as Eq. (9).

Precision $=($ Times that the images are correctly retrieved/ Total retrieval times) x 100\%

Similarly, supposing there are $\mathrm{N}$ candidate images similar with the retrieval image in the database, the socalled similarity with the retrieval image means their corresponding correlation coefficients are greater than a certain critical value. Here, we define the factor Recall to be the percentage of the candidate images similar with the retrieval image in the first $\mathrm{N}$ candidate image. The formula is expressed as Eq. (10).

Recall $=($ Number of retrieved similar image pieces /Number of total similar image pieces) $\times 100 \%$ (10)

Using these images of the medical image database described as above, we evaluate the performance of proposed scheme and the experimental results are 
shown in Table 1-5. Comparing Table 1 and Table 2, we can find the retrieval accuracy is better while the texture feature extracted from the feature image. Besides, we added the noises ranging from $\pm 8, \pm 16$ and \pm 32 , which are produced at random, to the retrieved images; the retrieval accuracy is shown as Tables 3 5; It is obvious that the similar images can be effectively retrieved by the features of shape and texture.

In summary, the experimental results show that retrieval accuracy using both shape and texture is better than only using one. Clearly, the performance of the proposed method is fast and efficient for medical image retrieval task

\section{CONCLUSIONS}

Different from the traditional image retrieval system that retrieves the single piece of static image one by one, the proposed retrieval strategy finds out the centroid image of the whole set of similar medical images, and then finds out the representative image aiming at the centroid images of different formats (compressed and uncompressed) to represent the set of medical images. From the experimental results, the proposed scheme can effectively achieve the retrieval tasks no matter aiming at the whole set of compressed and uncompressed images.

The future research will further make retrieval aiming at the medical images of local illness, i.e. liver tumor, connect the image collection system, image processing work station and image database system in

Table 1. Performance evaluation using the difference image features extracted from the whole image.

\begin{tabular}{|c|c|c|c|}
\hline ACCURACY & $\begin{array}{c}\text { SHAPE } \\
(8 X 8 P I X E L L S / B L O C K)\end{array}$ & $\begin{array}{c}\text { TFXTURF } \\
\text { (MF.AN } \\
\text { +VARIANCE) }\end{array}$ & $\begin{array}{c}\text { SHAPE } \\
+\end{array}$ \\
\hline precision & $98.97 \%$ & $100 \%$ & $100 \%$ \\
\hline recall & $93.54 \%$ & $68.31 \%$ & $98.51 \%$ \\
\hline
\end{tabular}

Table 2. Performance evaluation using the difference-image features extracted from the feature image.

\begin{tabular}{|c|c|c|c|}
\hline $\begin{array}{c}\text { ACCURACY } \\
\text { (FI:ATURF: } \\
\text { IMAGF) }\end{array}$ & $\begin{array}{c}\text { SHAPE } \\
(8 X 8 P I X E L S / B L O C K)\end{array}$ & $\begin{array}{c}\text { TEXTURE } \\
\text { (MEAN } \\
\text { +VARIANCF) }\end{array}$ & $\begin{array}{c}\text { SHAPF } \\
+ \\
\text { TEXTURE }\end{array}$ \\
\hline precision & $98.97 \%$ & $100 \%$ & $100 \%$ \\
\hline recall & $93.54 \%$ & $82.62 \%$ & $98.51 \%$ \\
\hline
\end{tabular}

Table 3. Performance evaluation using differenceimage features extracted from the median image of a set of medical images and extracted from the feature image of median image.

\begin{tabular}{|c|c|c|c|}
\hline Precision & $\begin{array}{c}\text { SHAPE } \\
(8 X 8 \text { PIXELS/BLOCK) }\end{array}$ & $\begin{array}{c}\text { TEXTURE } \\
\text { (MEAN } \\
\text { +VARIANCE) }\end{array}$ & $\begin{array}{c}\text { SHAPE } \\
+ \\
\text { TEXTURE }\end{array}$ \\
\hline $\begin{array}{c}\text { Median Image } \\
\begin{array}{c}\text { Feature image } \\
\text { of median } \\
\text { image }\end{array}\end{array}$ & $82.41 \%$ & $22.93 \%$ & $87.76 \%$ \\
\hline
\end{tabular}

Table 4. Performance evaluation using differenceimage features extracted from the whole image.

\begin{tabular}{|c|c|c|c|}
\hline NOISE RANGE & $\begin{array}{c}\text { SHAPE } \\
\text { (8X8PIXELS/BLOCK) }\end{array}$ & $\begin{array}{c}\text { TEXTURE } \\
\text { (MEAN } \\
\text { +VARIANCE) }\end{array}$ & $\begin{array}{c}\text { SHAPE } \\
\text { TEXTURE }\end{array}$ \\
\hline \pm 8 & $85.80 \%$ & $66.89 \%$ & $96.89 \%$ \\
\hline \pm 16 & $80.45 \%$ & $62.68 \%$ & $96.61 \%$ \\
\hline \pm 32 & $73.91 \%$ & $43.29 \%$ & $96.32 \%$ \\
\hline
\end{tabular}

Table 5. Performance evaluation using differenceimage features extracted from the feature image.

\begin{tabular}{|c|c|c|c|}
\hline $\begin{array}{c}\text { NOISE RANGE } \\
\text { (FEATURE) }\end{array}$ & $\begin{array}{c}\text { SHAPE } \\
\text { (8X8PIXELS/BLOCK) }\end{array}$ & $\begin{array}{c}\text { TEXTURE } \\
\text { (MEAN } \\
\text { +VARIANCE) }\end{array}$ & $\begin{array}{c}\text { SHAPE } \\
+ \\
\text { TEXTURE }\end{array}$ \\
\hline \pm 8 & $81.13 \%$ & $83.34 \%$ & $96.48 \%$ \\
\hline \pm 16 & $70.99 \%$ & $82.63 \%$ & $95.99 \%$ \\
\hline \pm 32 & $59.29 \%$ & $79.71 \%$ & $92.21 \%$ \\
\hline
\end{tabular}

the hospital, and combine all the various medical diagnosis image systems in the hospital, so as to make full use of the resources. When the doctor reads the electrical patient record, he can also read the relative images to further obtain the integral data of the patient. As a result, an effective and accepted diagnosis and treat environment is created for the doctors.

\section{ACKNOWLEDGEMENT}

This work is supported by Chang Gung Memorial Hospital under the contract CMRP1365. 


\section{REFERENCE}

1. Del Bimbo A. and Pala P., "Effective image retrieval using deformable templates", Pattern Recognition, vol. 3, pp. $120-124,1996$.

2. Patrice, B., Konik H. "Texture Similarity Queries and Relevance Feedback for Image Retrieval", Pattern Recognition, vol. 4, pp. 55 -58, 2000.

3. Kim B.Y., Kim H.J. and Jang S.J., "Image Retrieval Based on Color Coherence", Proceedings of the IEEE Region 10 Conference (TENCON 99), vol. 1, pp. $178-181,1999$.

4. Le Bourgeois F., "Content based image retrieval using gradient color fields", Pattern Recognition, vol. 1, pp. $1027-1030,2000$.

5. Daescu O., Kogge P.M. and Chen D.Z., "Parallel content-based image analysis on PIM processors", IEEE Workshop on Content-Based Access of Image and Video Libraries, pp. $73-77,1998$.

6. Lay J.A. and Ling Guan, "Image retrieval based on energy histograms of the low frequency DCT coefficients", IEEE International Conference, vol. 6, pp. $3009-3012,1999$.

7. Tolias Y., Panas S. and Tsoukalas L.H., "FSMIQ: fuzzy similarity matching for image queries", International Conference on Information Intelligence and Systems, pp. $249-254,1999$.
8. Kobayakwa M., Hoshi M. and Ohmori T, "Robust texture image retrieval using hierarchical correlation of wavelet coefficients", Pattern Recognition, vol. 3, pp. $391-396,2000$.

9. Yang C.C. and Chan M.C, "Color image retrieval based on textural and chromatic features", IEEE International Conference, vol. 4, pp.922-927, 1999.

10. Gevers T. and Smeulders A.W.M., "PicToSeek: Combing color and shape invariant features for image retrieval", IEEE Trans. Image Processing, vol. 91, pp. 102-119, Jan 2000.

11. Kwok-Wah Hung and Aw-Yong M, "A Contentbased Image Retrieval System Integrating Color,Shape and Spatial Analysis", IEEE International Conference on Systems Man and Cybernetics, vol.2, pp. 1481-1488, 2000.

12. Ruey-Feng Cheng, Wen-Jia Kuo and Hung-Chi Tsai, "Image Retrieval on Uncompressed and Compressed Domains", International Conference on Image Processing, vol. 2, pp. $546-549$, Jan. 2001.

13. K. Karadimitriou et al., "Centroid method for compressing sets of similar images", Pattern Recognition Letters, vol. 19, no. 7, pp.585-593, 1998. 